\title{
A comparison of desflurane consumption according to fresh gas flow
}

\author{
Deokkyu Kim ${ }^{1}$, Ji-Seon Son ${ }^{1}$, Jun Rae Lee ${ }^{2}$, Eunjoo Jang ${ }^{1}$, and Seonghoon Ko ${ }^{1}$ \\ ${ }^{1}$ Department of Anesthesiology and Pain Medicine, Chonbuk National University Medical School, ${ }^{2}$ Department of Oral and \\ Maxillofacial Surgery, Chonbuk National University Dentistry School, Jeonju, Korea
}

The consumption of volatile agents during general anesthesia can be altered by fresh gas flow (FGF), and it is known to be better to maintain an anesthetic effect with a lesser amount of volatile anesthetics because cost considerations, as long as it does not present a safety problem. A previous study reported that the consumption of sevoflurane and isoflurane was decreased in low FGF anesthesia, and the decreased amount was proportional to the FGF rate [1]. We hypothesized that desflurane consumption would proportionally depend on the FGF rate during anesthetic management in surgery.

After obtaining Institutional Review Board approval and informed consent, 42 patients, age 20-60 years and scheduled for oro-facial surgery, were randomly allocated to one of two groups: Group F1 received an FGF 1 L/min and Group F3 received an FGF $3 \mathrm{~L} / \mathrm{min}$. After arriving in the operating room, all patients were monitored by non-invasive blood pressure, pulse oximeter, electrocardiogram, Entropy, and Surgical Pleth Index (SPI) (GE Healthcare, Helsinki, Finland). Anesthesia induction was performed using propofol and rocuronium. After intubation, administration of $8 \%$ desflurane was initiated with an FGF $6 \mathrm{~L} / \mathrm{min}$ for the first five minutes and then the FGF was switched to 1 or $3 \mathrm{~L} / \mathrm{min}$ according to the assigned group. Anesthesia was maintained with only desflurane; no adjuvant drugs including nitrous oxide, opioids, intravenous anesthetics, and anti-hypertensive and vasoactive drugs. Blood pressure was maintained within $\pm 20 \%$ from the baseline value, which was obtained before anesthesia induction, and Entropy and SPI were kept below 50. If blood pressure, Entropy, or SPI were out of the mainte- nance range, the study was paused and the proper treatment was conducted for the patient's safety. Desflurane consumption was measured using an anesthesia machine (Avance $\mathrm{CS}^{\mathrm{TM}}{ }^{\mathrm{T}}$, GE Healthcare, Helsinki, Finland) at 5, 15, 30, and 60 minutes after intubation, when the administration of desflurane was initiated. The analysis was performed using SigmatPlot 12.5 (Systat Software Inc., San Jose, USA). Data were expressed as mean \pm SD or median (range; $25-75 \%$ ). The differences between two groups were analyzed by a t-test or Mann-Whitney rank sum test depending on the results of the normality and equal variance test. Statistical significance was considered at $\mathrm{P}<0.05$.

Five of 42 enrolled patients did not complete the study and were excluded from the statistical results; 18 patients in group F1 and 19 patients in group F3 completed as protocol. There was no statistical difference between the two groups in age, sex, height, weight, the time from intubation to skin incision, the type of surgery, hemodynamic parameters, and anesthetic depth. Desflurane consumption with a FGF $6 \mathrm{~L} / \mathrm{min}$ for the first five minutes after intubation was $15(13-15) \mathrm{ml}$ in the $\mathrm{F} 1$ group and $14(12-15) \mathrm{ml}$ in the $\mathrm{F} 3$ group, which were not statistically different $(\mathrm{P}=0.189)$. The desflurane consumption for one hour was $54(49-56) \mathrm{ml}$ in the F1 group and $94(86-105) \mathrm{ml}$ in the F3 group ( $\mathrm{P}<0.001$, Fig. 1$)$.

Although the FGF difference was three-fold (1 vs. $3 \mathrm{~L} / \mathrm{min}$ ) in this study, the desflurane consumption during one hour was less than twofold greater (54 vs. $94 \mathrm{ml}$ ). Weiskopt and Eger [2] calculated the desflurane consumption with various FGFs through simulation under the assumption that $1 \mathrm{MAC}$ was kept

Corresponding author: Seonghoon Ko, M.D., Ph.D., Department of Anesthesiology and Pain Medicine, Chonbuk National University Medical School, 20, Geonji-ro, Deokjin-gu, Jeonju 561-712, Korea. Tel: 82-63-250-1241, Fax: 82-63-250-1240, E-mail: shko@jbnu.ac.kr

() This is an open-access article distributed under the terms of the Creative Commons Attribution Non-Commercial License (http:// creativecommons.org/licenses/by-nc/3.0/), which permits unrestricted non-commercial use, distribution, and reproduction in any medium, provided the original work is properly cited. 


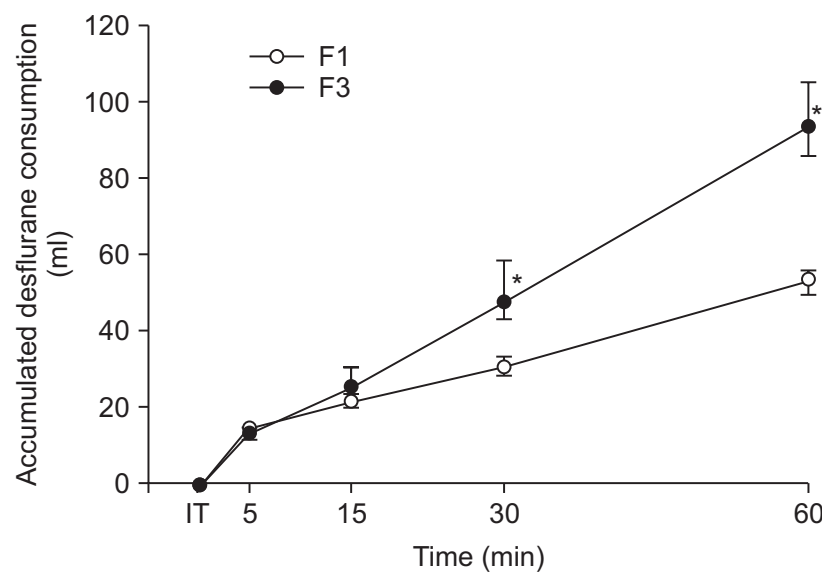

Fig. 1. The consumption of desflurane for 1 hour. Data are presented as median (range; $25-75 \%$ ). F1: group received a fresh gas flow of $1 \mathrm{~L} / \mathrm{min}$, F3: group received a fresh gas flow of $3 \mathrm{~L} / \mathrm{min}$, IT: intubation. $* \mathrm{P}<0.05$ compared with group F1 at 30 and 60 minutes after intubation, when the administration of desflurane was initiated.

constantly and found that desflurane consumption increased nearly twice - directly proportional to a twofold increase in FGF.

However, in our study, the desflurane consumption decreased less than a half with a threefold decrease in FGF. Several hypotheses to explain the cause can be postulated. First, a constant MAC was not maintained during anesthetic management in the present study. Proper anesthetic depth varies depending on the situation, and such factors including preparation for the operation after the anesthetic induction, a difference of surgical stimuli for the entire operation time, and individual variation can affect the depth. Second, relatively high concentrations of volatile anesthetics in low FGFs are required to reach the same anesthetic depth or MAC because the absolute amount of anesthetics to be delivered to the respiratory circuit of the anesthesia machine is decreased when FGF is decreased. In our study, a higher concentration of desflurane should be required in the F1 group compared to the F3 group, when the increasing of MAC was needed during the surgery. Third, although there was no statistically significant difference between 69 (61-78) $\mathrm{kg}$ in the F1 group and 63 (56-67) kg in the F3 group, the body weight in the F1 group was higher than that of the F3 group. Desflurane consumption may have increased more than expected due to the $10 \%$ body weight difference in the F1 group.

It is generally accepted that a low FGF is pharmacoeconomic due to the reduction in the consumption of volatile agents [1-3]. However, to reduce the FGF, a device that can monitor the respiratory gases is essential, but the monitoring device can increase the cost. When the FGF is low, the rebreathing fraction through the carbon dioxide absorbent is increased so that carbon monoxide production is increased by the degradation of anesthetics with the absorbent. The cost of carbon dioxide absorbent should also be considered.

There are some limitations to our study. Our study was conducted during anesthetic management for surgical stimuli, so it was difficult to maintain an equal anesthetic depth simultaneously in both groups. Second, because we did not record the dial setting of the vaporizer, there is no evidence that a higher dial setting was maintained in the F1 group compared to the F3 group. Third, there were no lower limits for Entropy and SPI, so there is a possibility of desflurane overdose. Fourth, the volatile agent consumption of Avance $\mathrm{CS} 2^{\mathrm{TM}}$ was calculated by a formula [4], not measured by the actual consumption amount. These factors may be a possible source of bias.

In conclusion, the desflurane consumption measured by Avance $\mathrm{CS} 2{ }^{\mathrm{TM}}$ for one hour was less in the FGF $1 \mathrm{~L} / \mathrm{min}$ than in the FGF $3 \mathrm{~L} / \mathrm{min}$, and the amount of desflurane saved was less than half during anesthetic management in surgery.

\section{References}

1. Kim DK, Choi JW, Son JS, Lim HS, Ko SH, Lee SK. A comparison of the consumption of inhaled anesthetics according to fresh gas flow and anesthetic circuits. Korean J Anesthesiol 2009; 56: 125-30.

2. Weiskopf RB, Eger EI 2nd. Comparing the costs of inhaled anesthetics. Anesthesiology 1993; 79: 1413-8.

3. Cotter SM, Petros AJ, Dore CJ, Barber ND, White DC. Low-flow anaesthesia. Practice, cost implications and acceptability. Anaesthesia 1991; 46: 1009-12.

4. Singaravelu S, Barclay P. Automated control of end-tidal inhalation anaesthetic concentration using the GE Aisys Carestation. Br J Anaesth 2013; 110: 561-6. 